\title{
Scalar-Field Amplitudes in Black-Hole Evaporation
}

\author{
A.N.St.J.Farley and P.D.D'Eath \\ Department of Applied Mathematics and Theoretical Physics, Centre for Mathematical Sciences, \\ University of Cambridge, Clarkson Road, Cambridge CB3 0WA, United Kingdom
}

\begin{abstract}
We consider the quantum-mechanical decay of a Schwarzschild-like black hole into almost-flat space and weak radiation at a very late time. That is, we are concerned with evaluating quantum amplitudes (not just probabilities) for transitions from initial to final states. In this quantum description, no information is lost because of the black hole. The Lagrangian is taken, in the first instance, to consist of the simplest locally supersymmetric generalization of Einstein gravity and a massless scalar field. The quantum amplitude to go from given initial to final bosonic data in a slightly complexified timeinterval $T=\tau \exp (-i \theta)$ at infinity may be approximated by the form const.exp $(-I)$, where $I$ is the (complex) Euclidean action of the classical solution filling in between the boundary data. Additionally, in a pure supergravity theory, the amplitude const.exp $(-I)$ is exact. Suppose that Dirichlet boundary data for gravity and the scalar field are posed on an initial spacelike hypersurface extending to spatial infinity, just prior to collapse, and on a corresponding final spacelike surface, sufficiently far to the future of the initial surface to catch all the Hawking radiation. Only in an averaged sense will this radiation have an approximately spherically-symmetric distribution. If the time-interval $T$ had been taken to be exactly real, then the resulting 'hyperbolic Dirichlet boundary-value problem' would, as is well known, not be well posed. Provided instead ('Euclidean strategy') that one takes $T$ complex, as above $(0<\theta \leq \pi / 2)$, one expects that the field equations become strongly elliptic, and that there exists a unique solution to the classical boundary-value problem. Within this context, by expanding the bosonic part of the action to quadratic order in perturbations about the classical solution, one obtains the quantum amplitude for weakfield final configurations, up to normalization. Such amplitudes are here calculated for weak final scalar fields.
\end{abstract}

\section{Introduction}

Since 1976, the most generally accepted option for the end-point of gravitational collapse to a black hole was that quantum coherence or information would be lost [1]. The gratifying change in opinion this past week [2] now makes it possible at last to begin publishing our late-1990's work on another option, namely, that information is not in fact lost, and that the end state is a combination of outgoing radiation states (see option 3 below). The present work began as a doctoral dissertation at Cambridge of one of the present authors [3] in 1997-2001 (the thesis was approved in summer 2002). This brief letter is an introductory sketch for numerous further papers contained in the thesis, which will provide detailed derivations of quantum amplitudes in black-hole evaporation. 
For simplicity, we fix attention in this paper on non-rotating black holes. In the semi-classical theory, a black hole of initial mass $M_{0}$ slowly radiates away its mass into predominantly massless particles, over a timescale $\sim\left(M_{0}\right)^{3}$ in Planck units $[4,5]$. Remarkably, at late retarded times, the emission depends only on the late-time quasi-stationary state of the 'black hole' and not on the precise details of its formation. The frequency spectrum has precisely the thermal character expected, with the Hawking temperature at infinity (units restored) given by

$$
T_{H}=\frac{\hbar c^{3}}{8 \pi G M_{0} k_{B}}
$$

where $k_{B}$ denotes Boltzmann's constant.

If one allows for the back-reaction of the emitted particles on the gravitational field, then, to a good approximation, one can model the black-hole evaporation with a sequence of quasi-stationary Schwarzschild solutions until the mass has nearly reached the Planck mass $[4,5]$. The Schwarzschild mass-loss rate balances the rate at which energy is radiated to infinity.

For the (ill-understood) very late stages of this evaporation, there are a number of conceivable options $[1,6-10]$. Some of the main options are:

1. The gravitational collapse could leave behind a naked singularity with negative mass (as there is no event horizon) which persists, thereby violating cosmic censorship;

2. A stable Planck-mass object might remain, containing the negative-energy quanta and stellar matter, so that the joint quantum state of the remnant and the emitted quanta would be pure;

3. The object might disappear completely, but all information about black-hole configurations and any locally conserved quantities might still escape to infinity. That is, particle correlations would be restored, so that the final state of the quantum field would be pure;

4. The object might disappear completely, taking with it the information about the stellar matter, negative-energy quanta and any quantities not coupled to long-range fields, so that the final state would be mixed. The nature of the final radiation in this instance, and in option 3 above, is still to be uncovered.

This paper focusses on options 3 and 4 and probabilities or quantum amplitudes associated with such outcomes. Below, we will describe how the total amplitude of (probable) weak-field final configurations can be computed from the imaginary part of the Lorentzian action, which is quadratic in the field perturbations in the linearised theory. The computation of such amplitudes for late-time scalar perturbations is the main result to be reported in this paper.

For simplicity, we take the bosonic matter to be described by a massless scalar field $\phi$. The full Lagrangian might, in principle, be more complicated, containing also appropriate fermionic fields and (say) Maxwell or Yang-Mills fields. Indeed, although we shall not study fermionic fields in this paper, it will be simplest (see Sec. 2) to assume that the Lagrangian is that of [ ], consisting of supermatter - a complex massless scalar field and spin - $1 / 2$ 
partner - coupled to $N=1$ supergravity, consisting of Einstein gravity with gravitino partner. In a linearised approximation, we expand the massless scalar field and the metric around a background classical field configuration, in which $\phi$ is taken to be real, describing a (bosonic) spherically symmetric 'matter' collapse, in which only the scalar field acts as a matter source for gravity. For both scalar and gravitational fields, 'Dirichlet boundary data' (see below) are specified on a (edgeless) spacelike hypersurface $\Sigma_{i}$ at an early time - roughly the moment of collapse - and on a final late-time spacelike hypersurface $\Sigma_{f}$, long after the black hole has evaporated. The location of $\Sigma_{f}$ will be such as to register all the evaporated radiation. Both surfaces extend to spatial infinity.

To fix one's physical intuition, it may be imagined that the energy density of the initial scalar configuration is extremely diffuse, such that almost all of the mass is distributed over radii vastly greater than the 'Schwarzschild radius' $2 M_{0}$. Further, the initial time-variation of the scalar and the gravitational fields can be taken to be extremely slow.

Away from the black hole, typical wavelengths of perturbation modes are small in comparison with the local radius of curvature of the space-time - the characteristic length scale over which the background space-time changes significantly. Prior to the black hole's final disappearance, however, typical wavelengths of perturbation modes which will contribute significantly to radiation in the late-time quantum treatment are comparable in magnitude to the black-hole radius. Correspondingly, one finds that the radiating Schwarzschild-like Vaidya metric [11-13] models, to a good approximation, the background space-time of an evaporating black hole at late times, outside the central strong-field region. Such a semi-classical description will need to be joined onto a comparable treatment of the more non-linear central regions.

Nevertheless, there is an intrinsically 'non-classical' character to this formulation, since wave-like boundary-value problems are well known to be badly posed [14]. Analogously, the Uncertainty Principle forbids the simultaneous specification of a dynamical variable and its conjugate momentum - precisely the data specified in a Cauchy problem. The price that we pay for our boundary-value formulation may be a lack of existence and/or uniqueness in the solution of the boundary-value problem, in the case of a real time-interval $T$ at infinity; this is intimately connected with the occurrence of singularities. But experience of other simpler such 'hyperbolic boundary-value problems', such as the flat-space wave equation, leads one to expect that there will be a (complex) classical solution, typically unique, provided that we rotate the proper-time separation at infinity, $T$, infinitesimally into the complex, à la Feynman, and indeed that a real Riemannian solution will be reached when $T$ is rotated by a phase of $\pi / 2$ to a Euclidean proper distance $\tau=i T$. There is a substantial mathematical theory of such strongly elliptic Dirichlet boundary-value problems in the complex, with existence, uniqueness and regularity properties corresponding to the more familiar real elliptic case [15].

\subsection{Existence for the full Einstein-scalar system}

A variety of spherically symmetric boundary-value examples in the complexified version of general relativity, coupled to a massless scalar field, are being worked out, and will then be published. One would like to show that (three-dimensional) asymptotically flat 
initial and final boundary data (Dirichlet data) for the spherically-symmetric gravitational and scalar fields can be specified, together with (in the first instance) a purely Euclidean 'time-interval' $|T|$, such that there is a smooth classical Riemannian infilling solution of the coupled Einstein/massless scalar field equations, agreeing with the given Dirichlet boundary data. This requirement is perhaps not unreasonable, since the coupled Riemannian field equations are 'elliptic modulo gauge', and elliptic equations tend to smooth out boundary data. Further, that, as the time-interval-at-infinity $T$ is rotated back in the complex, towards the Lorentzian region, by a phase less than $\pi / 2$, while holding fixed the Dirichlet data on the initial and final surfaces, then the full complex classical solution varies smoothly with the phase, as does the classical action.

In particular, if the scalar-field part of the Dirichlet data is chosen to be sufficiently strong, then the corresponding Lorentzian-signature solution, reached by a phase rotation by $\pi / 2$, will describe collapse to a black hole. Only as one approaches a phase of $\pi / 2$ would the classical solution become singular. Of course, this would be in accord with the singularity theorems of general Lorentzian-signature relativity [16]. This view of strongly elliptic versus singular Lorentzian-signature solutions also shows further the insight of Feynman's $+i \epsilon$ prescription.

Note that the mass $M$ of the classical 'space-time' is a functional of the boundary data, including the possibly complex time-at-infinity $T[17,18]$. It has been found in the above examples that $M$ is negative when $T=-i \tau$, for $\tau$ real, corresponding to a real Riemannian solution. The geometry is regular at the origin $r=0$ of spherical symmetry (and elsewhere) and the Riemannian solution lives on a portion of Euclidean space $\mathbb{R}^{4}$. One does not encounter the different topology of the Riemannian positive-mass Schwarzschild solution $[19,20]$.

As described in $[17,18,21]$, each of the intrinsic spatial metrics $h_{i j I}$ and $h_{i j F}$ on $\Sigma_{I}$ and $\Sigma_{F}$ will have an intrinsic Arnowitt-Deser-Misner mass $M_{A D M}[22,23]$, measured by the usual $1 / r$ fall-off rate of the spatial metric near spatial infinity. But (see Sec.4.4 of [18]) in general, for the classical infilling metric $g_{\mu \nu}$, the obvious surfaces of constant time will be emitted in such a way that the mass $M$ of the 4-dimensional geometry differs from the '3-dimensional mass' $M_{A D M}$. It is this which allows for the possibility that the mass $M$ of the classical geometry may depend on the time-separation $T$ at infinity, as well as on the bounding 3 -metrics $h_{i j I}, h_{i j F}$, as in the previous paragraph.

Note also: it is essential that the 3-metrics prescribed on $\Sigma_{I}$ and $\Sigma_{F}$ should each have the same intrinsic ADM mass $M_{A D M}$. Otherwise $[17,18]$, any classical infilling 'space-time' will have these 3 -surfaces badly embedded near spatial infinity.

In the more general non-spherical black-hole problem to be studied in the rest of this paper, the slight complexification of $T$ induces an imaginary part in the total (Lorentzian) action, which leads (below) to the quantum amplitudes and probabilities needed to examine options 3 and 4 above.

\section{The quantum amplitude}

Consider first quantum field theory for gravity coupled to bosonic and fermionic fields. In particular, take the case in which non-trivial bosonic data are specified on the 
boundary surfaces, while for simplicity the fermionic boundary data are taken to be zero. The 'Euclidean' quantum amplitude to go between the prescribed initial and final data is given formally by a Feynman path integral. Naively, disregarding the problem of infinities, one would expect the amplitude to have the asymptotic form

$$
\text { Amp } \sim\left(A_{0}+\hbar A_{1}+\hbar^{2} A_{2}+\ldots\right) \exp \left(-I_{B} / \hbar\right) .
$$

Here $I_{B}$ is the real classical 'Euclidean' action of a Riemannian solution of the coupled Einstein/bosonic-matter classical field equations. [For simplicity, we assume that there is a unique classical solution.] Further, $I_{B}$ and the loop terms $A_{0}, A_{1}, A_{2}, \ldots$ depend in principle on the boundary data. For our case of matter coupled to Einstein gravity, $I_{B}, A_{0}, A_{1}, \ldots$ also obey differential constraints connected with the local coordinate invariance of the theory, and with any other local invariances such as gauge invariance [24].

In particular, when the theory is also invariant under local supersymmetry, and when the fermionic boundary data are now allowed to be non-zero, the above semi-classical expansion (2) becomes extremely simple. For example, for $N=1$ supergravity, one has $[18,25]$ :

$$
\text { Amp } \sim A_{0} \exp (-I / \hbar) .
$$

This is not just a formal expression, as in Eq.(2), but an exact statement, freed of infinities. The 'one-loop factor' $A_{0}$ is in fact a constant, while $I$ denotes the action of the classical solution, which includes both bosonic and fermionic parts. Thus, in this case, the classical action is all that is needed for the quantum computation. A corresponding situation arises with ultra-high-energy collisions, whether between black holes [26], in particle scattering [27], or in string theory [28]. For more complicated field theories possessing local supersymmetry, such as supergravity coupled to supermatter [29,30], quantum amplitudes may well still be meaningful since free of divergences. Their semi-classical expansion is, however, expected to have the more general form (2).

The difficulty of assigning meaning to quantum amplitudes in field theories which contain Einstein gravity and other lower-spin fields, but which are not invariant under local supersymmetry, might also encourage one to turn to string theory. But it is again true in string theory that only for the superstring models, which are invariant under local supersymmetry transformations, does one have meaningful finite amplitudes [31,32].

In the asymptotically-flat, spatially- $\mathbb{R}^{3}$ context here, the purely Riemannian case above corresponds to a time-separation at spatial infinity of the usual rotated form $T=$ $-i \tau$, where $\tau$ is a positive imaginary-time separation. Consider further the locally supersymmetric case, but take only the bosonic data to be non-zero (real) on the initial and final surfaces. Suppose that there is a unique infilling Riemannian classical solution. Following the standard route, one can study the (now complex) bosonic amplitude (2) or (3) as $T$ is rotated through angles $\theta$ from $\theta=\pi / 2$ to $\theta=+\epsilon$, with

$$
T=\exp (-i \theta) \tau
$$

Provided that there continues to exist a (now complex) bosonic classical solution to the Dirichlet problem, as $\theta>0$ decreases from $\pi / 2$ towards zero, the expression (2) or (3) 
should still continue to give the form of the (analytic) quantum amplitude. In particular, this would occur if strong ellipticity held for the coupled Einstein/bosonic-matter field equations.

\section{The classical action and amplitude for weak perturbations}

\subsection{The approximate space-time metric}

The classical background bosonic fields, the metric $g_{\mu \nu}$ and (real) scalar field $\phi$, are each taken to have a 'large' time-dependent spherically symmetric part and a 'small' perturbative part, which can be expanded out in terms of sums over tensor, vector and scalar harmonics [33-39]. Each harmonic is weighted by a function of the time- and radial coordinates $(t, r)$. The 'large' or 'background' Lorentzian space-time metric can be put in the form $[40,41]$ :

$$
d s^{2}=-e^{b(t, r)} d t^{2}+e^{a(t, r)} d r^{2}+r^{2}\left(d \theta^{2}+\sin ^{2} \theta d \varphi^{2}\right) .
$$

The 'background' scalar field is denoted by $\Phi(t, r)$. The classical spherically-symmetric part of the Einstein and scalar field equations will be as given in [40] ('Lorentzian') or [41] (Riemannian) for an exactly spherically-symmetric set of fields, except for an additional effective energy-momentum contribution $\left\langle T_{\mu \nu}^{E F F}\right\rangle$, resulting from local space-time averaging of the contribution to the Einstein equations of terms quadratic and higher in the combined perturbations $[42,43]$. It will be assumed that $\left\langle T_{\mu \nu}^{E F F}\right\rangle$ is defined in such a way as to be spherically symmetric.

\subsection{Scalar field - harmonic decomposition}

Now consider purely bosonic weak classical perturbations around this dynamic background. For simplicity of exposition, consider a 'small' real scalar perturbation, denoted by $\phi^{(1)}$, where $\phi=\Phi+\phi^{(1)}$. Because of the approximate spherical symmetry, one may expand the perturbation $\phi^{(1)}$ in the form

$$
\phi^{(1)}(t, r, \theta, \varphi)=\frac{1}{r} \sum_{\ell=0}^{\infty} \sum_{m=-\ell}^{\ell} Y_{\ell m}(\Omega) R_{\ell m}(t, r)
$$

Here, $Y_{\ell m}(\Omega)$ denotes the $(\ell, m)$ spherical harmonic of [44]. The boundary conditions on the radial functions $R_{\ell m}(t, r)$ as $r \rightarrow 0$ follow from the regularity at $r=0$. Recall that, in the strongly elliptic case, all fields are analytic in the interior of the large cylindrical boundary formed by the initial and final surfaces together with a surface at large $r$.

The perturbed scalar wave equation at late times,

$$
\nabla^{\mu} \nabla_{\mu} \phi^{(1)}=0
$$

with respect to the background geometry, leads to the $(\ell, m)$ mode equation

$$
\left[e^{(b-a) / 2} \partial_{r}\right]^{2} R_{\ell m}-\left(\partial_{t}\right)^{2} R_{\ell m}-\left(\frac{1}{2}\right)\left[\partial_{t}(a-b)\right]\left(\partial_{t} R_{\ell m}\right)-V_{\ell}(t, r) R_{\ell m}=0
$$


where

$$
V_{\ell}(t, r)=\frac{e^{b(t, r)}}{r^{2}}\left[\ell(\ell+1)+\frac{2 m(t, r)}{r}\right]
$$

is real and positive in the 'Lorentzian' case. Here, $m(t, r)$ is defined by $\exp [-a(t, r)]=$ $1-[2 m(t, r)] / r$. This potential $V_{\ell}(t, r)$ generalises the well-known massless-scalar effective potential in the exact Schwarzschild space-time [23,39], which vanishes at the event horizon $\{r=2 M\}$ and at infinity, and has a peak near $\{r=3 M\}$.

There is, of course, an analogous harmonic decomposition of the weak gravitationalwave perturbations about the nearly-spherically-symmetric background [33-39].

\subsection{The classical action}

After detailed calculation [3,45], one finds that the classical Lorentzian action for a classical bosonic solution to our model, including the quadratic-order contribution of weak perturbations, can be written as

$$
S_{\text {class }}\left[h_{i j}^{(1)}, \phi^{(1)}\right]=\frac{1}{32 \pi} \int_{\Sigma_{f}} d^{3} x \pi^{(1) i j} h_{i j}^{(1)}+\frac{1}{2} \int_{\Sigma_{f}} d^{3} x \phi^{(1)} \pi_{\phi^{(1)}}-M T .
$$

Here, for simplicity, it is assumed that the only non-zero (prescribed) boundary perturbations are in the intrinsic spatial metric $h_{i j}^{(1)}$ and in the real part of the scalar field $\phi^{(1)}$ on the final surface $\Sigma_{f}$. The corresponding perturbations in the initial boundary data $h_{i j}^{(1)}$ and $\phi^{(1)}$ on $\Sigma_{i}$ have been taken to be zero. Of course, one could easily include them also. Here $\pi^{(1) i j}$ is the linearized perturbation of the momentum canonically conjugate to the intrinsic spatial $g_{i j}$, given in [23]. Also,

$$
\pi_{\phi^{(1)}}=n^{(0) \mu} \nabla_{\mu} \phi^{(1)}
$$

is the momentum canonically conjugate to $\phi^{(1)}$. Here, $n^{(0) \mu}$ is the future-directed unit time-like normal vector to the three-surface (here $\Sigma_{f}$ ), in the 'Lorentzian' case. Further, in Eq.(10), $M$ is the '4-dimensional mass' of the 'space-time', as in Sec.1.1. Typically, $M$ will differ from the ADM mass $M_{A D M}$ found from the intrinsic 3 -metrics $h_{i j I}, h_{i j F}$.

\subsection{The quantum amplitude for scalar perturbations on the final surface}

On the initial surface $\Sigma_{i}$, we take the initial data such that there is a quasi-stationary Schwarzschild-like background. On or near the final surface $\Sigma_{f}$, long after evaporation, one again expects a nearly-Schwarzschild background; the characteristic time-scale over which the background geometry varies is very much greater than the period of a typical wave. In such cases, the radial wave functions $R_{\ell m}(t, r)$ can be decomposed harmonically with respect to $t$, and can be conveniently normalised (below). Hence, near $\Sigma_{f}$ say, one can write

$$
\phi^{(1)}=\frac{1}{r} \sum_{\ell m} \int_{0}^{\infty} d k a_{k \ell m} R_{k \ell m}(t, r) Y_{\ell m}(\Omega)
$$


where the $\left\{a_{k \ell m}\right\}$ are real quantities. Spherical symmetry implies that, on $\Sigma_{f}$,

$$
R_{k \ell m}(t, r)=R_{k \ell}(r)
$$

is independent of $m$, where $R_{k \ell}(r)$ is a real function. Note [see below, Eq.(20)] that the allowed values of $k$ are discrete, labelled by an integer $n=0,1,2, \ldots$, and are given by $k=k_{n}=n \pi /|T|$.

We describe briefly the normalisation of the $R_{k \ell}(r)$. With the notation $\tilde{r}=k r$, the boundary conditions of regularity as $r \rightarrow 0$ imply

$$
R_{k \ell}(r) \propto r j_{\ell}(\tilde{r}) \sim \operatorname{const.} \tilde{r}^{\ell+1},
$$

where $j_{\ell}(\tilde{r})$ are (real) spherical Bessel functions [46]. For a background geometry which is nearly Schwarzschild at large radius, one also has

$$
R_{k \ell}(r) \sim z_{k \ell} e^{i k r_{S}^{*}}+\bar{z}_{k \ell} e^{-i k r_{S}^{*}}
$$

as $r \rightarrow \infty$, where, for large $r$,

$$
r_{S}^{*} \sim r+2 M \log \left(\left(\frac{r}{2 M}\right)-1\right)
$$

is the Regge-Wheeler 'tortoise' coordinate $[23,33]$. Here the $\left\{z_{k \ell}\right\}$ are dimensionless complex coefficients which are determined by the regularity above at $r=0$, subject to suitable normalisation [3]. The $z_{k \ell}$ are related to the Bogoliubov coefficients $[47,48]$, thus making contact with the original formulation of black-hole evaporation $[4,5]$. The detailed relations are described in the fuller works $[3,45]$.

Given the above information in a neighbourhood of the final surface $\Sigma_{f}$, one can compute the classical scalar contribution to the Lorentzian action $S_{\text {class }}\left[h_{i j}^{(1)}, \phi^{(1)}\right]$ of Eq.(10), in the case that the time-interval $T=\tau e^{-i \theta}$ at infinity is rotated slightly into the complex. This classical scalar contribution will in general be complex. The Euclidean action is then defined via

$$
-I_{\text {class }}=i S_{\text {class }} \text {. }
$$

In the limit that $\theta \rightarrow 0$ (while keeping $\theta$ strictly positive), one finds that the (bosonic) quantum amplitude has the form (say, in the case of a locally supersymmetric Lagrangian):

$$
\text { Amp } \sim \text { const. } \exp \left(-I_{\text {class }}\right)
$$

apart from possible loop corrections [Eq.(2)] which will only come into play at or above the Planck energy. This amplitude has a (computable) oscillating part in each mode, multiplied by a product of Gaussians

$$
|A m p| \propto \exp \left(-\frac{4 \pi^{3}}{|T|^{2}} \sum_{n=1}^{\infty} \sum_{\ell=0}^{\infty} \sum_{m=-\ell}^{\ell} n\left|z_{n \ell}\right|^{2}\left|a_{n \ell m}\right|^{2}\right)
$$


in the coordinates $\left\{a_{k \ell m}\right\}$ of Eq.(12) for the perturbed final scalar data. Here, because of the large-but-finite time interval $|T|$, the eigen-frequencies are of the form

$$
k=k_{n}=\frac{n \pi}{|T|} .
$$

\section{Comments and further work}

By using a field-based amplitude in quantum gravity and by rotating the time interval $T$ at infinity slightly into the complex: $T \rightarrow \tau e^{-i \theta}$, one expects to have a strongly elliptic classical boundary-value problem. For weak wave fields of different spin at the final late-time boundary $\Sigma_{f}$, after the black-hole evaporation, one can compute the quantum amplitudes for different final configurations as $\exp \left(-I_{\text {class }}\right)$, up to an overall factor. An explicit example of this for the scalar-field perturbations of our model was given in Sec.3. [Full details will be given in [45] and further papers.] Analogous 'Gaussian' results for the spin-1 Maxwell field and for spin-2 gravitational-wave perturbations are contained in [3] and will also be submitted; similarly for the fermionic case.

The late-time radiation can be viewed either in a field or in a particle representation, and has the usual interpretation in terms of thermal Hawking radiation.

Work is also under way on numerical solution of the complexified boundary-value problem for the coupled Einstein-scalar field equations, with real data posed on the initial and final three-surfaces, and a complex time-interval $T$ specified at spatial infinity, as considered above. This requires generalisation of the original Riemannian Einstein/scalar numerical boundary-value work of [41]. This should help one to understand, for example, the nature of any burst of radiation which might originate in the late stages of complexified 'collapse'.

It would also be very interesting if similar methods could be applied in the case that the spatial bounding surfaces are not just two copies of $\mathbb{R}^{3}$.

\section{Aknowledgements}

We are grateful to an unnamed referee for suggestions which led to substantial improvements in the Letter.

\section{References}

[1] S.W.Hawking, Phys. Rev. D 14, 2460 (1976).

[2] S.W.Hawking, communication, GR17 Conference, Dublin, 18-24 July (2004).

[3] A.N.St.J.Farley, 'Quantum Amplitudes in Black-Hole Evaporation', Cambridge Ph.D. dissertation, approved 2002 (unpublished).

[4] S.W.Hawking, Nature (London) 248, 30 (1974).

[5] S.W.Hawking, Commun. Math. Phys. 43, 199 (1975).

[6] S.W.Hawking, Commun. Math. Phys. 87, 395 (1982).

[7] S.W.Hawking, 'Boundary Conditions of the Universe' in Astrophysical Cosmology, Proceedings of the Study Week on Cosmology and Fundamental Physics, eds. H.A.Brück, G.V.Coyne and M.S.Longair. Pontificia Academiae Scientarium: Vatican City, 48, 563 (1982). 
[8] R.M.Wald, in Quantum Theory of Gravity, ed. S.Christensen, (Adam Hilger, Bristol) 160 (1984).

[9] P.Hájiček and W.Israel, Phys. Lett. A 80, 9 (1980).

[10] J.Bardeen, Phys. Rev. Lett. 46, 382 (1981).

[11] A.Das, M.Fischler and M. Roček, Phys.Lett.B 69, 186 (1977).

[11] P.C.Vaidya, Proc. Indian Acad. Sci. A33, 264 (1951).

[12] H.Stephani, D.Kramer, M.A.H. MacCallum, C.Hoenselaers and E.Herlt, Exact solutions of Einstein's field equations, 2nd. edition, (Cambridge University Press, Cambridge) (2003).

[13] A.Krasiński, Inhomogeneous Cosmological Models, (Cambridge University Press, Cambridge) (1997).

[14] P.R.Garabedian, Partial Differential Equations, (Wiley, New York) (1964).

[15] W.McLean, Strongly Elliptic Systems and Boundary Integral Equations, (Cambridge University Press, Cambridge) (2000).

[16] S.W.Hawking and G.F.R.Ellis, The large scale structure of space-time, (Cambridge University Press, Cambridge) (1973).

[17] P.D.D'Eath, Phys.Rev. D24, 811 (1981).

[18] P.D.D'Eath, Supersymmetric Quantum Cosmology, (Cambridge University Press, Cambridge) (1996).

[19] J.B.Hartle and S.W.Hawking, Phys.Rev.D13, 2188 (1976).

[20] S.W.Hawking, 'The path-integral approach to quantum gravity', in General Relativity. An Einstein Centenary Survey, eds. S.W.Hawking and W.Israel (Cambridge University Press, Cambridge) (1979).

[21] J.A.Wheeler, 'Superspace and the Nature of Quantum Geometrodynamics' p.303, in Battelle Rencontres, ed. C.M.DeWitt and J.A.Wheeler (W.A.Benjamin, New York) (1968). [22] R.Arnowitt, S.Deser and C.W.Misner, 'Dynamics of General Relativity', in Gravitation: An Introduction to Current Research, ed. L.Witten (Wiley, New York) (1962).

[23] C.W.Misner, K.S.Thorne and J.A.Wheeler, Gravitation, (Freeman, San Francisco) (1973).

[24] P.A.M.Dirac, Lectures on Quantum Mechanics, (Academic Press, New York) (1965).

[25] P.D.D'Eath, 'What local supersymmetry can do for quantum cosmology', in The Future of Theoretical Physics and Cosmology, eds. G.W.Gibbons, E.P.S.Shellard and S.J.Rankin (Cambridge University Press, Cambridge) 693 (2003).

[26] P.D.D'Eath, Black Holes: Gravitational Interactions, (Oxford University Press, Oxford) (1996).

[27] G. 't Hooft, Phys. Lett. B 198, 61 (1987).

[28] S. Giddings, 'Black holes at accelerators', in The Future of Theoretical Physics and Cosmology, eds. G.W.Gibbons, E.P.S.Shellard and S.J.Rankin (Cambridge University Press, Cambridge) 278 (2003).

[29] J.Wess and J.Bagger, Supersymmetry and Supergravity, 2nd. edition, (Princeton University Press, Princeton) (1992).

[30] P.D.D'Eath, 'Loop amplitudes in supergravity by canonical quantization', in Fundamental Problems in Classical, Quantum and String Gravity, ed. N.Sánchez (Observatoire de Paris) 166 (1999), hep-th/9807028. 
[31] M.B.Green, J.H.Schwarz and E.Witten, Superstring Theory, vols. 1 and 2, (Cambridge University Press, Cambridge) (1987).

[32] M.B.Green, 'A brief description of string theory' in The Future of Theoretical Physics and Cosmology eds. G.W.Gibbons, E.P.S. Shellard and S.J.Rankin (Cambridge University Press, Cambridge) 473 (2003).

[33] T.Regge and J.A.Wheeler, Phys. Rev. 108, 1063 (1957).

[34] J.Mathews, J. Soc. Ind. Appl. Math. 10, 768 (1962).

[35] J.N.Goldberg, A.J.MacFarlane, E.T.Newman, F.Rohrlich and E.C.G.Sudarshan, J. Math. Phys. 8, 2155 (1967).

[36] C.V.Vishveshwara, Phys. Rev. D 1, 2870 (1970).

[37] F.J.Zerilli, Phys. Rev. D 2, 2141 (1970).

[38] W.L.Burke, J. Math. Phys. 12, 401 (1971).

[39] J.A.H.Futterman, F.A.Handler and R.A.Matzner, Scattering from Black Holes, (Cambridge University Press, Cambridge) (1988).

[40] M.W.Choptuik, 'Critical Behaviour in Massless Scalar-Field Collapse', in Approaches to Numerical Relativity, ed. R.d'Inverno, (Cambridge University Press, Cambridge) (1992).

[41] P.D.D'Eath and A.Sornborger, Class. Quantum Grav. 15, 3435 (1998).

[42] D.Brill and J.B.Hartle, Phys. Rev. 135, 1327 (1964).

[43] R.Isaacson, Phys. Rev. 166, 1263, 1272 (1968).

[44] J.D.Jackson, Classical Electrodynamics, (Wiley, New York) (1975).

[45] A.N.St.J.Farley and P.D.D'Eath, 'Quantum Amplitudes in Black-Hole Evaporation', paper being submitted.

[46] M.Abramowitz and I.A.Stegun, Handbook of Mathematical Functions, (Dover, New York) (1964).

[47] N.D.Birrell and P.C.W.Davies, Quantum fields in curved space, (Cambridge University Press, Cambridge) (1982).

[48] V.P.Frolov and I.D.Novikov, Black Hole Physics, (Kluwer Academic, Dordrecht) (1998). 